\title{
Expression of proteins associated with epithelial-mesenchymal transition in esophageal squamous cell carcinoma
}

\author{
TIAN ZHANG ${ }^{1,2 *}$, HAO LI $^{1 *}$, YANG ZHANG ${ }^{1}$, PEI WANG $^{1}$, HUIJIE BIAN ${ }^{1}$ and ZHI-NAN CHEN ${ }^{1}$ \\ ${ }^{1}$ Department of Cell Biology, National Translational Science Center for Molecular Medicine, \\ Fourth Military Medical University, Xi'an, Shaanxi 710032; ${ }^{2}$ Department of Laboratory Medicine and Pathology, \\ The People's Liberation Army 59 Central Hospital, Kaiyuan, Yunnan 661600, P.R. China
}

Received July 5, 2016; Accepted November 20, 2017

DOI: $10.3892 / \mathrm{ol} .2017 .7701$

\begin{abstract}
Cluster of differentiation 147 (CD147), pituitary tumor transforming gene (PTTG) and CD44v6 are proteins involved in the epithelial-mesenchymal transition (EMT). To investigate the prognostic value of CD147 and PTTG, and CD44v6 expression in esophageal squamous cell carcinoma (ESCC), tissue microarray specimens from 76 patients with ESCC were evaluated by immunohistochemistry staining and scored by intensity and proportion of positive areas. Expression levels of CD147, PTTG and CD44v6 were higher in tumor tissues than in matched adjacent tissues. CD147 expression was positively associated with lymph node metastasis $(\mathrm{P}=0.025)$ and American Joint Committee on Cancer (AJCC) system clinical grades $(\mathrm{P}=0.037)$. CD147 expression was positively correlated with the expression levels of PTTG $(\mathrm{R}=0.369$; $\mathrm{P}=0.001)$ and $\mathrm{CD} 44 \mathrm{v} 6(\mathrm{R}=0.320 ; \mathrm{P}=0.005)$. In addition, Kaplan-Meier analysis indicated that positive expression of CD147, PTTG and CD44v6 was significantly associated with poor overall survival times $(\mathrm{P}=0.045, \mathrm{P}=0.014$ and $\mathrm{P}=0.027$, respectively). Patients exhibiting CD147-PTTG co-expression, CD147-CD44v6 co-expression and CD147-PTTG-CD44v6 triple-positive expression had the poorest overall survival rates. In conclusion, the expression of EMT-associated proteins, including CD147, PTTG and CD44v6, was significantly associated with poor survival in ESCC and these novel targets may serve as potential biomarkers for anticancer therapies.
\end{abstract}

Correspondence to: Professor Zhi-Nan Chen or Professor Huijie Bian, Department of Cell Biology, National Translational Science Center for Molecular Medicine, Fourth Military Medical University, 169 Changle West Road, Xi'an, Shaanxi 710032, P.R. China

E-mail: znchen@fmmu.edu.cn

E-mail: hjbian@fmmu.edu.cn

*Contributed equally

Key words: cluster of differentiation 147, pituitary tumor transforming gene, cluster of differentiation 44 variant 6 , esophageal squamous cell carcinoma, prognosis, epithelial-mesenchymal transition

\section{Introduction}

Esophageal carcinoma is one of the most aggressive malignancies with poor prognosis and has been defined as two major histologic types: Esophageal squamous cell carcinoma (ESCC) and esophageal adenocarcinoma (1). ESCC is the fourth most frequently diagnosed cancer and the fourth leading cause of cancer-associated mortality in China (2). Lymph node metastasis is one of the biggest challenges in treatment and survival of this type of cancer. Further therapeutic improvements are required and a focus on targeted therapy is warranted. This has led to increased interest in the prognostic and therapeutic value of tumor biomarkers that are known to serve a key role in carcinogenesis and progression.

Evidence indicates that epithelial-mesenchymal transition (EMT) serves a key role in a variety of diseases, including cardiovascular disease and cancer (3). EMT is of importance during the processes of tumorigenesis and metastasis; EMT has been reported to reduce the adhesion and increase the motility of epithelial tumor cells (4). The underlying mechanism is essential to early-phase cancer metastasis. Numerous signaling pathways, including transforming growth factor- $\beta$ (TGF- $\beta$ ) and Notch, participate in the progression of EMT and the target genes are responsible for activation of the mesenchymal phenotype (5).

Cluster of differentiation 147 (CD147), also known as basigin, is highly expressed on the surface of carcinoma cells, but not on that of normal cells (6). CD147 is a heavily glycosylated type I transmembrane glycoprotein; its overexpression of CD147 was significantly associated with various malignant tumors (7). In addition, a report demonstrated that CD147 served an important role in EMT and regulated diverse signaling pathways (8). Pituitary tumor transforming gene (PTTG) is an oncogene that is highly expressed in a variety of tumor tissues (9). The overexpression of PTTG stimulated the expression of matrix metalloproteinase-2 and enhanced EMT process in oral squamous cell carcinoma (10). CD44v6, a splice variant of CD44, is highly associated with tumor invasion and metastasis and is reported to be a key biomarker for certain types of metastatic cancer (11). A prior study reported that CD44v6 contributed to autophagy, EMT and the activation of numerous pathways in colon cancer (12). However, the association between EMT and ESCC is not fully understood. 
Further investigation to identify EMT-associated proteins in ESCC is required; these proteins may be associated with adverse patient prognosis and an improved understanding of them could contribute to the development of therapeutic strategies for patients with ESCC.

In the present study, the expression levels of CD147, PTTG and CD44v6 in ESCC tissues were analyzed; the correlation between the expressions of these EMT-associated molecules was also assessed. Furthermore, Kaplan-Meier analysis was performed to investigate the prognostic value of CD147, PTTG and CD44v6 in patients with ESCC.

\section{Materials and methods}

Pathological specimens. A tissue microarray, purchased from Shanghai Outdo Biotech Co., Ltd. (cat. no. HEso-Squ172Sur-01; Shanghai, China) contained 86 cases totally from patients who underwent surgery at hospitals between July 2006 and September 2008. Tissue specimens were collected from 64 males and 22 females, with a median age of 65 years (range 41-81). Excluding those who were lost to follow-up or lacked pathological information, the samples from 76 patients were enrolled in the present study, including 57 male patients and 19 female patients with a median age of 65 years ranging from 41 to 81 years. Additionally, 72 paired adjacent normal tissues in the same microarray were used for comparison in the same manner. All patients had a single tumor and no distant metastasis and the clinical stages were classified by the American Joint Committee on Cancer system (AJCC) (13).

Immunohistochemical staining. The staining procedure was performed by standard streptavidin-horseradish peroxidase complex method. The slides were dried in an incubator at $65^{\circ} \mathrm{C}$ overnight, and following deparaffinization via $100 \%$ xylene for $15 \mathrm{~min}$ and hydration using $100 \%$ ethanol for $5 \mathrm{~min}, 95 \%$ ethanol for $2 \mathrm{~min}, 80 \%$ ethanol for $2 \mathrm{~min}$ and $75 \%$ ethanol for $2 \mathrm{~min}$. Tissue sections were incubated with $3 \%$ hydrogen peroxide in methanol at room temperature for $30 \mathrm{~min}$ to block endogenous peroxidase activity. Briefly, the anti-PTTG (1:50 dilution; cat. no. 12575-1-AP; ProteinTech Group, Inc., Chicago, IL, USA), anti-CD44v6 (pre-diluted; cat. no. 2M-0052; OriGene Technologies, Inc., Beijing, China), and anti-CD147 (CD147 Diagnostic kit; cat. no. CL001-01; Jiangsu Pacific-Meinuoke Biopharmaceutical Co., Ltd., Jiangsu, China) antibodies were applied and incubated at $4^{\circ} \mathrm{C}$ overnight, followed by washing with PBS. The sections were incubated with a secondary antibody (ready-to-use kit; cat. no. SP9000; goat anti-mouse IgG; OriGene Technologies, Inc.), for $30 \mathrm{~min}$ at room temperature. Signals were developed with 3,3-diaminobenzine substrate (1:200; cat no. ZLI-9018; OriGene Technologies, Inc.) for 2 min and counterstained with hematoxylin (ready-to-use; cat. no. BA-4041; Baso Diagnostic, Inc.) for $10 \mathrm{~min}$ at room temperature.

Evaluation of immunohistochemical staining. Protein expression determined by immunohistochemical staining was evaluated using an Olympus microscope (Olympus Corporation, Tokyo, Japan). The sections were carefully diagnosed under light microscope and five random fields of view were examined at magnification, x200 and x400. Staining for these biomarkers was classified by the staining intensity and the percentage of positive tumor cells. The percentage of positively stained cells was counted for each tissue section. According to the degree of staining, the expression was defined as follows: - (negative), + (weak intensity; $5-30 \%$ positive tumor cell), ++ (moderate intensity; $30-70 \%$ positive tumor cell) and +++ (strong intensity; $>70 \%$ positive tumor cell).

Statistical analysis. Associations between immunohistochemical staining patterns and clinicopathological parameters were evaluated by $\chi^{2}$ tests. The correlation between the expression of these biomarkers was analyzed by Spearman's correlation coefficient. Kaplan-Meier statistical analysis and a log-rank test were used to assess the survival curve and compare between subgroups. Statistical analyses were performed by SPSS 19.0 (IBM Corp., Armonk, NY, USA). P<0.05 was considered to indicate a statistically significant difference.

\section{Results}

Characteristics of the selected patients with ESCC. The age of patients ranged between 41 and 81-years, the mean age was 65 . Of the patients, there were 57 males and 19 females. By the time of the final follow-up in September 2013, 52 patients had succumbed to mortality, during a median follow-up time of 30.5 months (range, 0-85.0 months).

Association between EMT-associated molecule expression and clinicopathological parameters. In the present study, CD147 expression was detected in 69 samples (90.8\%) and was enriched on the surface of the cancer cells. However, in adjacent normal tissues, staining for CD147 antibody was almost negative (data not shown). Representative histological pictures are presented in Fig. 1. Statistical analysis revealed that increased CD147 expression levels were associated with patient sex $(\mathrm{P}=0.003)$, AJCC clinical stage $(\mathrm{P}=0.037)$ and lymph node metastasis $(\mathrm{P}=0.025$; Table I). PTTG expression was detected in 52 cancer tissues $(68.4 \%)$ and was also observed in the nucleus and plasma of cancer cells (Fig. 1). Overexpression of PTTG was significantly associated with histological grade $(\mathrm{P}=0.035$; Table I), but not with lymph node metastasis or AJCC clinical stage. Furthermore, the staining for CD44v6 (Fig. 1), mainly in the cell membrane, appeared in 59 carcinoma tissues $(77.6 \%)$. However, the overexpression of CD44v6 within tumor tissues was not associated with histological grade, lymph node metastasis and AJCC clinical grade.

Correlation between CD147, PTTG and CD44v6 expression. In the present study, the correlation between the expression of CD147, PTTG and CD44v6 was assessed using Spearman's correlation analysis. CD147 expression was significantly correlated with the expression of PTTG (Table II) and CD44v6 (Table III). There was also a significant correlation between PTTG and CD44v6 expression $(\mathrm{R}=0.527, \mathrm{P}=0.001$; Table IV).

Effects of CD147, PTTG and CD44v6 expression on OS rate in patients with ESCC. Kaplan-Meier curves were generated 


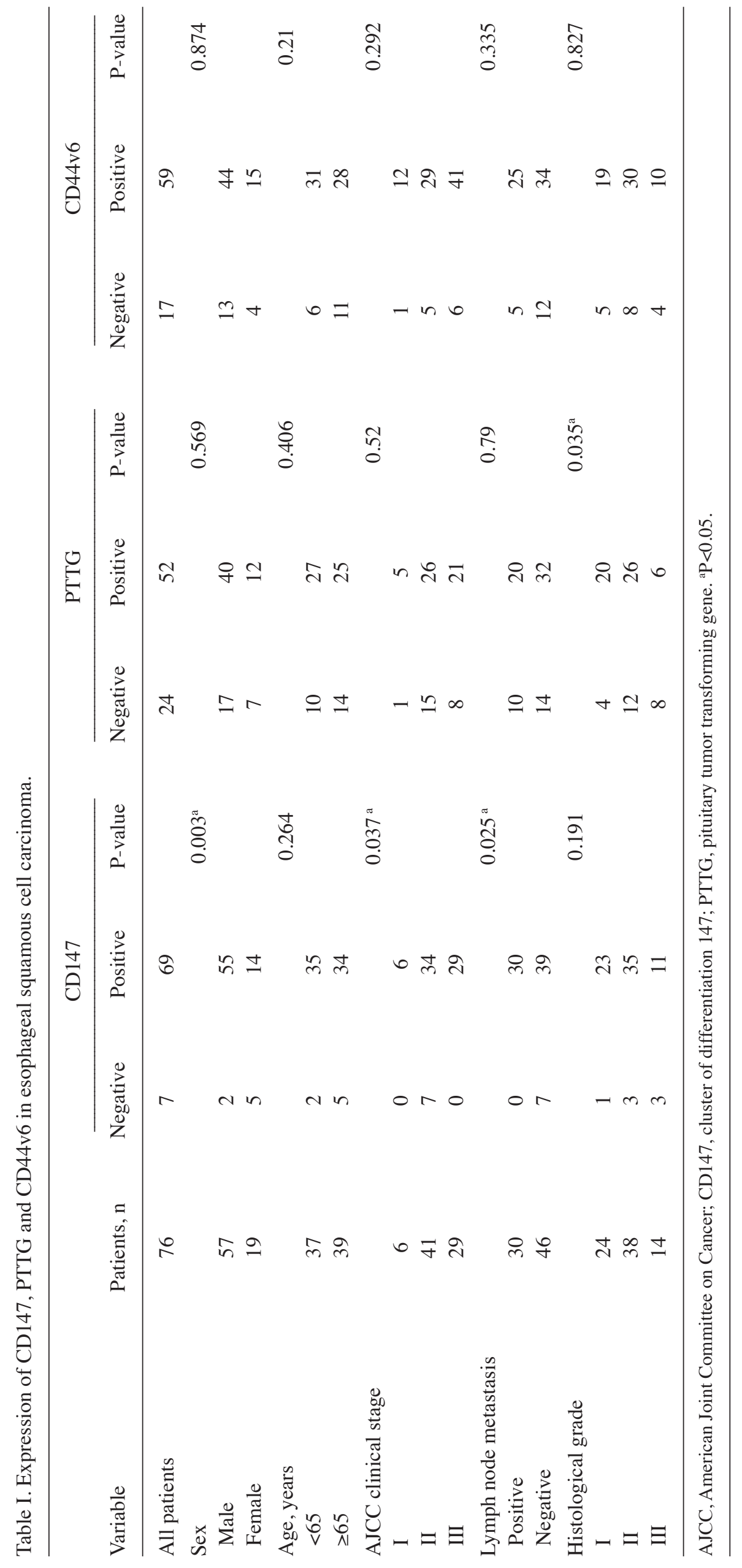


Table II. Correlation between CD147 and PTTG expression.

\begin{tabular}{|c|c|c|c|c|c|c|c|}
\hline & & \multicolumn{4}{|c|}{ PTTG } & \multicolumn{2}{|c|}{ Spearman's correlation } \\
\hline & & - & + & ++ & +++ & R-value & P-value \\
\hline \multirow[t]{4}{*}{ CD147 } & - & 7 & 0 & 0 & 0 & 0.369 & 0.001 \\
\hline & + & 12 & 24 & 3 & 4 & & \\
\hline & ++ & 4 & 6 & 6 & 0 & & \\
\hline & +++ & 1 & 5 & 4 & 0 & & \\
\hline
\end{tabular}

CD147, cluster of differentiation 147; PTTG, pituitary tumor transforming gene; -, negative staining; +, weak staining; ++, moderate staining; +++ , strong staining.

Table III. Correlation between CD147 and CD44v6 expression.

\begin{tabular}{|c|c|c|c|c|c|c|c|}
\hline & & \multicolumn{4}{|c|}{ CD44v6 } & \multicolumn{2}{|c|}{ Spearman's correlation } \\
\hline & & - & + & ++ & +++ & R-value & P-value \\
\hline \multirow[t]{3}{*}{ CD147 } & - & 4 & 2 & 1 & 0 & 0.320 & 0.005 \\
\hline & + & 9 & 17 & 11 & 6 & & \\
\hline & ++ & 2 & 4 & 2 & 8 & & \\
\hline
\end{tabular}

CD147, cluster of differentiation 147; -, negative staining; +, weak staining; ++, moderate staining; +++, strong staining.

Table IV. Correlation between PTTG and CD44v6 expression.

\begin{tabular}{|c|c|c|c|c|c|c|c|}
\hline & & \multicolumn{4}{|c|}{ PTTG } & \multicolumn{2}{|c|}{ Spearman's correlation } \\
\hline & & - & + & ++ & +++ & $\mathrm{R}$-value & $\mathrm{P}$-value \\
\hline \multirow[t]{4}{*}{ CD44v6 } & - & 13 & 4 & 0 & 0 & 0.527 & $<0.001$ \\
\hline & + & 7 & 14 & 2 & 2 & & \\
\hline & ++ & 2 & 10 & 5 & 0 & & \\
\hline & +++ & 2 & 7 & 6 & 2 & & \\
\hline
\end{tabular}

CD44v6, cluster of differentiation 44 variant 6; PTTG, pituitary tumor transforming gene; -, negative staining; +, weak staining; ++, moderate staining; +++, strong staining.

for patients with ESCC categorized patients according to the expression of CD147, PTTG and CD44v6. Patients negative for CD147 expression exhibited longer OS rate than patients with CD147 positive expression, a difference that was statistically significant (Fig. 2A). The results also indicated that the duration of survival was significantly associated with positive PTTG expression (Fig. 2B). Additionally, the patients with positive CD44v6 expression experienced a poorer survival rate (Fig. 2C). As CD147 expression was significantly associated with the expression of PTTG and CD44v6, patients were divided into subgroups according to the combined expression of these EMT-associated markers to detect whether the prediction of ESSC prognosis was more accurate. Regarding co-expression, patients with positive staining for CD147 and PTTG demonstrated lower OS rates (Fig. 2D). Furthermore, the combination of CD147 and CD44v6 expression suggested that the overall survival of these subgroups had a statistical difference (Fig. 2E). Positive expression of CD147, PTTG and CD44v6 was associated with the poorest patient prognosis (Fig. 2F).

\section{Discussion}

In the present study, the expression levels of CD147, PTTG and CD44v6 protein were investigated in a tissue microarray of samples from 76 patients with ESCC. The results demonstrated that the overexpression of these three proteins was significantly associated with various pathological characteristics and may be considered as a predictor of shorter OS times in patients with ESSC.

CD147 is overexpressed in various tumor cells and may promote tumor invasion and lymph node metastasis (14). 
CD147
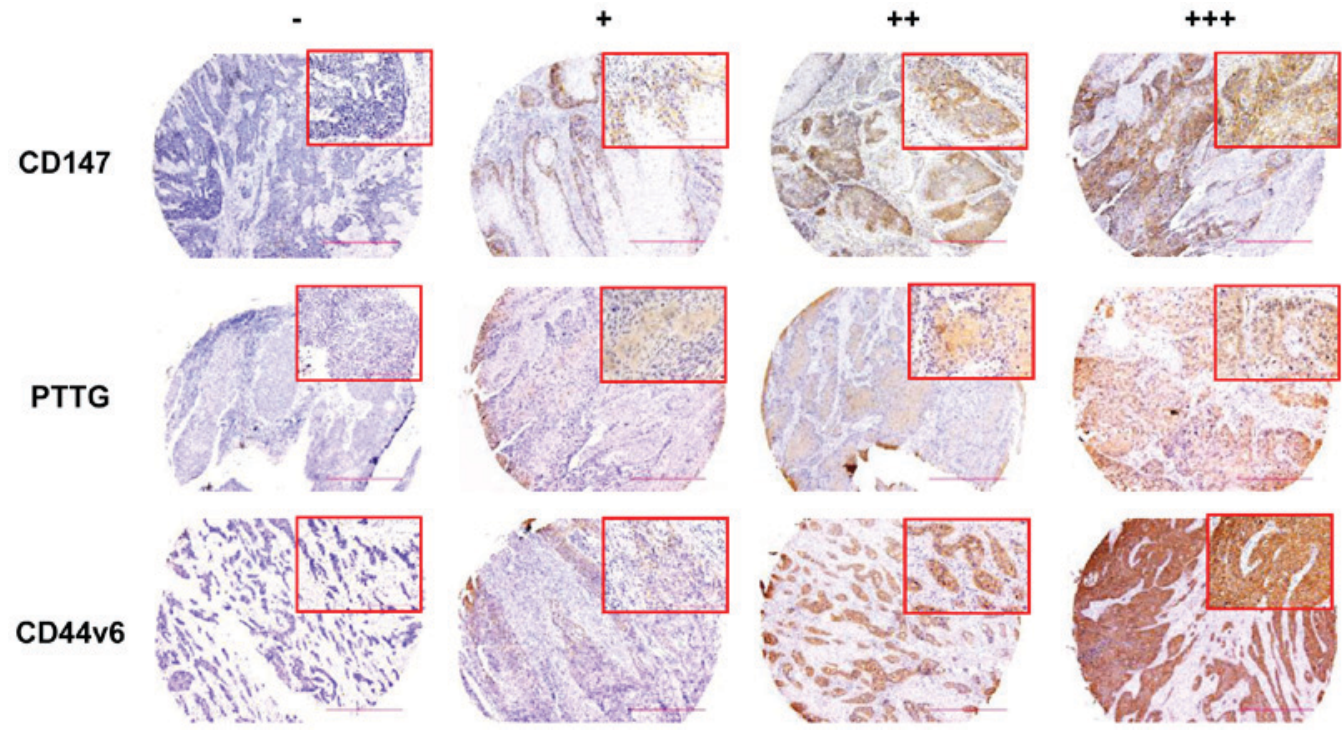

Figure 1. Immunohistochemical photomicrographs of CD147, PTTG and CD44v6 in tissue samples from patients with esophageal squamous cell carcinoma. The expression of CD147, PTTG and CD44v6 was classified as - (negative staining), + (weak staining), ++ (moderate staining) or +++ (strong staining); magnifications, x100 (main image) and x400 (inset). CD147, cluster of differentiation 147; PTTG, pituitary tumor transforming gene.
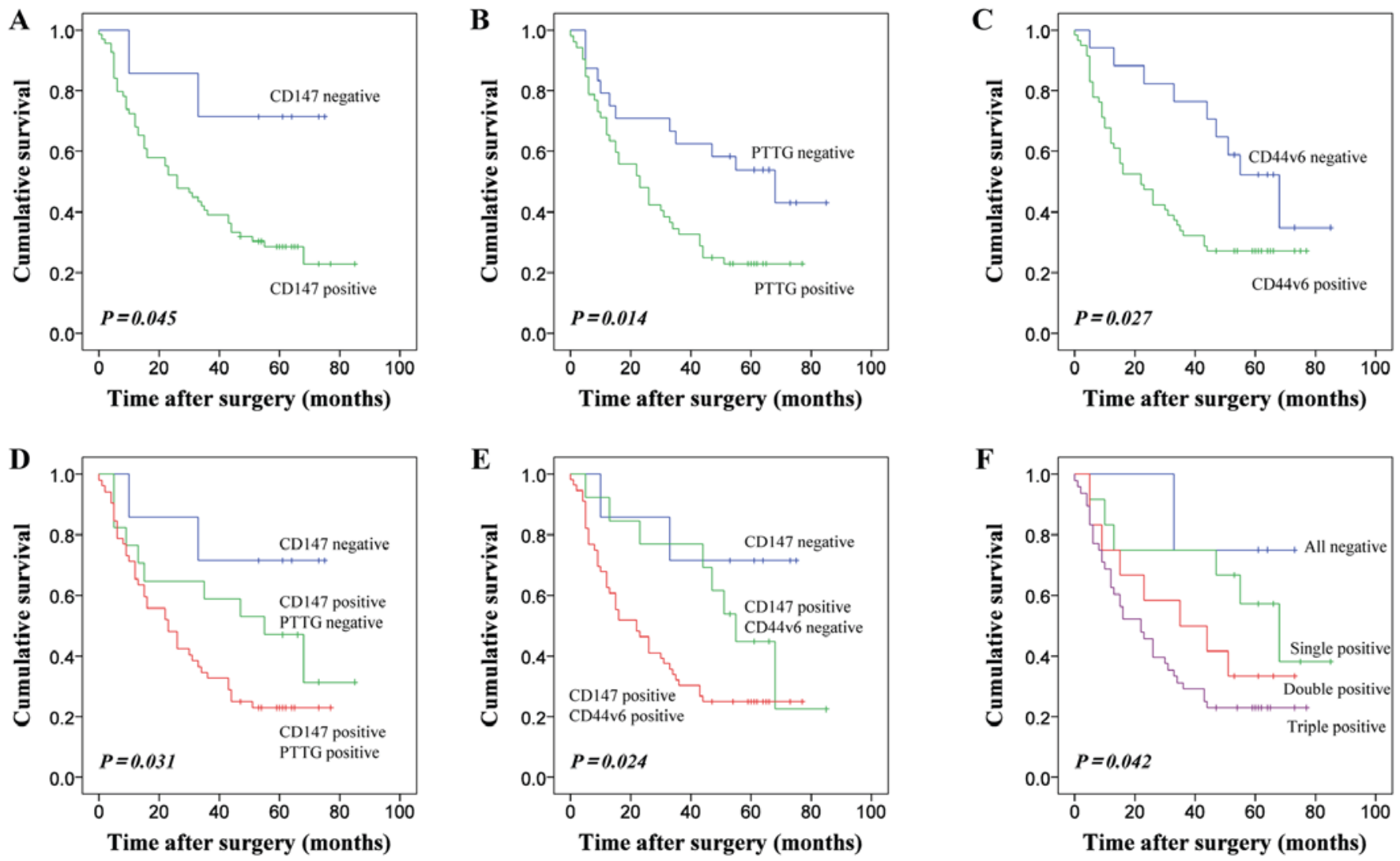

Figure 2. Kaplan-Meier analysis of CD147, PTTG and CD44v6 expression for patients with esophageal squamous cell carcinoma. (A) Patients with positive CD147 expression had poorer OS rate than those with negative expression ( $\mathrm{P}=0.045)$. (B) Patients with positive PTTG expression had poorer OS times than those with negative expression $(\mathrm{P}=0.014)$. (C) Patients with positive $\mathrm{CD} 44 \mathrm{v} 6$ expression had a poorer $\mathrm{OS}$ rate than those with negative expression ( $\mathrm{P}=0.027)$. (D) Association between the combination of CD147 and PTTG expression with overall survival ( $\mathrm{P}=0.031)$. (E) Association between the combination of $\mathrm{CD} 147$ and $\mathrm{CD} 44 \mathrm{v} 6$ expression with overall survival $(\mathrm{P}=0.024)$. (F) Association between the combination of $\mathrm{CD} 147$, and $\mathrm{CD} 44 \mathrm{v} 6$ expression with overall survival $(\mathrm{P}=0.042)$. CD147, cluster of differentiation 147; PTTG, pituitary tumor transforming gene.

As a target gene of the TGF- $\beta$ signaling pathway, CD147 induces EMT and may be a potential target for the treatment of HCC (15). In the present study, CD147 was reportedly increased in human ESCC. The overexpression of CD147 was associated with advanced with the AJCC clinical stages and lymph node metastasis, consistent with previous data reported 
by Zhu et al (16). Furthermore, when these features were subjected to Kaplan-Meier analysis, the expression of CD147 was associated with lower OS rates.

PTTG is an oncogene that is regulated by the $\beta$-catenin signaling pathway in human ESCC (17). PTTG has been suggested to accelerate the induction of EMT in lung cancer by regulating integrin $\alpha_{v} \beta_{3}$ and adhesion-complex proteins, such as talin, paxillin, vinculin and actinin (18). EMT is also induced by PTTG in human ovarian epithelial cancer cells via the regulation of TGF- $\beta$ and E-cadherin (19). A previous study demonstrated that the overexpression of PTTG may contribute to the malignant progression of ESCC and serve as an independent prognostic factor of OS time in ESCC (20). PTTG enhances the metastatic potential of breast cancer cells by inducing CD147 via the activation of the focal adhesion kinase/protein kinase $\mathrm{B} /$ mechanistic target of rapamycin signaling pathway (21). The findings of the present study demonstrated that the level of PTTG expression was associated with the duration of survival and the histological grade of the disease. However, the poorer OS times of individuals with double-positive expression of CD147 and PTTG indicated that these proteins may collectively participate in the regulation of tumorigenesis and metastasis in ESCC; thus, further investigation into the underlying mechanism is required.

CD44v6 expression is associated with higher tumor stage and metastatic potential in numerous types of tumors. In colorectal cancer, high expression levels of CD44v6 revealed a significant inverse correlation with E-cadherin expression and a positive correlation with vimentin expression (22). However, the role of CD44v6 expression was controversial in certain other tumor types, including oral squamous cell carcinoma (23). The results of the present study indicated that the overexpression of CD44v6 was a useful marker for patient prognosis; the combination of CD44v6 and CD147 was associated with reduced OS rate.

EMT has been considered to be a critical step that promotes the early phase of cancer metastasis. EMT-activating signaling pathways and downstream proteins are responsible for promoting EMT (24). In a recent study, PTTG promoted invasion in a human breast cancer cell line by upregulating CD147 (17). In addition, CD147, as a novel upstream activator of signal transducer and activator of transcription 3 (STAT3), interacts with CD44 and serves as a critical role in the development of pancreatic cancer (25). Furthermore, the present study revealed that patients with tumors that concurrently expressed these three markers exhibited an inferior OS rate. This result indicated that the increased expression levels of EMT-associated proteins in ESCC may substantially alter the prognosis of patients. In addition, the identification of distinct prognostic subgroups can be beneficial for the accuracy of ESSC prognosis in the clinical practice.

In conclusion, the overexpression of CD147, PTTG and CD44v6, individually, was associated with the development of ESSC and was significantly associated with OS rate, which indicated that these proteins are potential biomarkers for prognosis. Additionally, the significant correlation between CD147, PTTG and CD44v6 expression indicated that these molecules may collectively participate in the regulation of EMT-associated signaling pathways in ESCC. CD147, PTTG and CD44v6 may be considered as potential targets for the development of anticancer therapies.

\section{Acknowledgements}

The present study was supported by the National Natural Science Foundation of China (grant no. 31571434) and the National Basic Research Program of China (grant no. 2015CB553701)

\section{References}

1. Pennathur A, Gibson MK, Jobe BA and Luketich JD: Oesophageal carcinoma. Lancet 381: 400-412, 2013.

2. Lin Y, Totsuka Y, He Y, Kikuchi S, Qiao Y, Ueda J, Wei W, Inoue $\mathrm{M}$ and Tanaka $\mathrm{H}$ : Epidemiology of esophageal cancer in Japan and China. J Epidemiol 23: 233-242, 2013.

3. Chua KN, Poon KL, Lim J, Sim WJ, Huang RY and Thiery JP: Target cell movement in tumor and cardiovascular diseases based on the epithelial-mesenchymal transition concept. Adv Drug Deliv Rev 63: 558-567, 2011.

4. De Craene B and Berx G: Regulatory networks defining EMT during cancer initiation and progression. Nat Rev Cancer 13: 97-110, 2013

5. Lamouille S, Xu J and Derynck R: Molecular mechanisms of epithelial-mesenchymal transition. Nat Rev Mol Cell Biol 15: 178-196, 2014.

6. Weidle UH, Scheuer W, Eggle D, Klostermann S and Stockinger H: Cancer-related issues of CD147. Cancer Genomics Proteomics 7: 157-169, 2010.

7. Toole BP: Emmprin (CD147), a cell surface regulator of matrix metalloproteinase production and function. Curr Top Dev Biol 54: 371-389, 2003.

8. Ru NY, Wu J, Chen ZN and Bian H: HAb18G/CD147 is involved in TGF- $\beta$-induced epithelial-mesenchymal transition and hepatocellular carcinoma invasion. Cell Biol Int 39: 44-51, 2015.

9. Vlotides G, Eigler T and Melmed S: Pituitary tumor-transforming gene: Physiology and implications for tumorigenesis. Endocr Rev 28: 165-186, 2007.

10. Zhang E, Liu S, Xu Z, Huang S, Tan X, Sun C and Lu L: Pituitary tumor-transforming gene 1 (PTTG1) is overexpressed in oral squamous cell carcinoma (OSCC) and promotes migration, invasion and epithelial-mesenchymal transition (EMT) in SCC15 cells. Tumour Biol 35: 8801-8811, 2014.

11. Orian-Rousseau V: CD44, a therapeutic target for metastasising tumours. Eur J Cancer 46: 1271-1277, 2010.

12. Lv L, Liu HG, Dong SY, Yang F, Wang QX, Guo GL, Pan YF and Zhang XH: Upregulation of CD44v6 contributes to acquired chemoresistance via the modulation of autophagy in colon cancer SW480 cells. Tumour Biol 37: 8811-8824, 2016.

13. Rice TW, Gress DM, Patil DT, Hofstetter WL, Kelsen DP and Blackstone EH: Cancer of the esophagus and esophagogastric junction-Major changes in the American Joint Committee on Cancer eighth edition cancer staging manual. CA Cancer J Clin 67: 304-317, 2017.

14. Yan L, Zucker S and Toole BP: Roles of the multifunctional glycoprotein, emmprin (basigin; CD147), in tumour progression. Thromb Haemost 93: 199-204, 2005.

15. Wu J, Ru NY, Zhang Y, Li Y, Wei D, Ren Z, Huang XF, Chen ZN and Bian H: HAb18G/CD147 promotes epithelial-mesenchymal transition through TGF- $\beta$ signaling and is transcriptionally regulated by Slug. Oncogene 30: 4410-4427, 2011.

16. Zhu S, Li Y, Mi L, Zhang Y, Zhang L, Gong L, Han X, Yao L, Lan M, Chen Z and Zhang W: Clinical impact of HAb18G/CD147 expression in esophageal squamous cell carcinoma. Dig Dis Sci 56: 3569-3576, 2011

17. Zhou C, Liu S, Zhou X, Xue L, Quan L, Lu N, Zhang G, Bai J, Wang Y, Liu Z, et al: Overexpression of human pituitary tumor transforming gene (hPTTG), is regulated by beta-catenin/TCF pathway in human esophageal squamous cell carcinoma. Int J Cancer 113: 891-898, 2005.

18. Shah PP, Fong MY and Kakar SS: PTTG induces EMT through integrin $\alpha \mathrm{V} \beta 3$-focal adhesion kinase signaling in lung cancer cells. Oncogene 31: 3124-3135, 2012. 
19. Shah PP and Kakar SS: Pituitary tumor transforming gene induces epithelial to mesenchymal transition by regulation of Twist, Snail, Slug, and E-cadherin. Cancer Lett 311: 66-76, 2011.

20. Zhang J, Yang Y, Chen L, Zheng D and Ma J: Overexpression of pituitary tumor transforming gene (PTTG) is associated with tumor progression and poor prognosis in patients with esophageal squamous cell carcinoma. Acta Histochem 116: 435-439, 2014.

21. Gao H, Zhong F, Xie J, Peng J and Han Z: PTTG promotes invasion in human breast cancer cell line by upregulating EMMPRIN via FAK/Akt/mTOR signaling. Am J Cancer Res 6 : 425-439, 2016

22. Saito S, Okabe H, Watanabe M, Ishimoto T, Iwatsuki M, Baba Y, Tanaka Y, Kurashige J, Miyamoto Y and Baba H: CD44v6 expression is related to mesenchymal phenotype and poor prognosis in patients with colorectal cancer. Oncol Rep 29: 1570-1578, 2013.
23. Kunishi M, Kayada Y and Yoshiga K: Down-regulated expression of CD44 variant 6 in oral squamous cell carcinomas and its relationship to regional lymph node metastasis. Int J Oral Maxillofac Surg 26: 280-283, 1997.

24. Nieto MA, Huang RY, Jackson RA and Thiery JP: EMT: 2016. Cell 166: 21-45, 2016

25. Li L, Tang W, Wu X, Karnak D, Meng X, Thompson R, Hao X, Li Y, Qiao XT, Lin J, et al: HAb18G/CD147 promotes pSTAT3-mediated pancreatic cancer development via CD44s. Clin Cancer Res 19: 6703-6715, 2013

(c) (i) $($ This work is licensed under a Creative Commons Attribution-NonCommercial-NoDerivatives 4.0 International (CC BY-NC-ND 4.0) License. 